\title{
Mucosal vaccination with phage-displayed tumour antigens identified through proteomics-based strategy inhibits the growth and metastasis of 4T1 breast adenocarcinoma
}

\author{
MOHSEN SHADIDI, DAG SØRENSEN, ANNE DYBWAD, GRO FURSET and MOULDY SIOUD \\ Department of Immunology, Molecular Medicine Group, Institute for Cancer Research, \\ Rikshospitalet-Radiumhospitalet Medical Center, Montebello, N-0310 Oslo, Norway
}

Received July 27, 2007; Accepted September 10, 2007

\begin{abstract}
Cancer immunotherapy relies on the identification and characterization of tumour antigens that can be recognized by effector T cells. Here, we used a proteomics-based approach to identify tumour antigens recognized by serum antibodies from patients with breast cancer. Specific reactivity against a set of spots was identified and their identity was revealed by MALDI-TOF peptide mass fingerprinting. They include disintegrin and metalloprotease 10, aldolase A, B-ATPase F1, heat shock protein 27 , deaminase, pyruvate dehydrogenase protein $\mathrm{X}$ component, and Vimentin. Western blot analysis using recombinant proteins expressed in E. coli confirmed the specific reactivity with patient sera. Several tumour antigens were expressed on the surface of the T7 phage and shown to trigger specific immune responses in BALB/c mice following oral immunisation. Furthermore, these immune responses inhibited tumour growth and metastasis of the 4T1 mammary adenocarcinoma cell line. Collectively, the present data indicate that proteomics-based strategy can identify tumour antigens whose surface display on phages or bacteria can provide an effective strategy for mucosal cancer vaccines. In addition, arrayed phage-displayed tumour antigens could be useful as a serum-based screening test for the detection of several tumour antigens.
\end{abstract}

\section{Introduction}

Despite progress in cancer therapy, breast cancer still accounts for approximately $30 \%$ of all cancer in females. Therefore, there is a need for the identification of therapeutic, prognostic and/or diagnostic biomarkers in order to improve detection time and treatment. The probing of humoral immune responses

Correspondence to: Dr M. Sioud, Department of Immunology, Institute for Cancer Research, Rikshopitalet-Radiumhospitalet Medical Center, Montebello, N-310 Oslo, Norway

E-mail:mosioud@ulrik.uio.no

Key words: immunotherapy, proteomics, mammary adenocarcinoma, mucosal vaccination, tumour antigens in patients with cancer is expected to facilitate the identification of biomarkers and the development of effective cancer vaccines. In this respect, a number of different cloning strategies have been developed over the past 2 decades to identify tumour antigens recognized by $\mathrm{T}$ cells (1). Among the described strategies, the serological analysis of recombinant tumour cDNA expression libraries (SEREX) has made it possible to identify tumour antigens without the need of establishing $\mathrm{T}$ cell lines (2). Despite the number of tumour antigens identified by SEREX (3), the sensitivity of this method is low because it relies on only one screening step. To circumvent this limitation, we have developed a new strategy based upon the use of phage display libraries and shown that specific immune responses imprinted in patient sera can be probed by this method (4). In principle, the phage display approach is far superior to the one-step screening method because sensitivity and selectivity are extremely high when selecting though iterative powerful enrichment steps. When peptides or protein phage libraries were biopanned on breast cancer sera, IgG antibody-reacting peptides or proteins were identified $(5,6)$. The antibody reactivity to some of the selected $\mathrm{B}$ cell epitopes correlated with patient prognosis. In addition, we have found that immune responses that are associated with epitope spreading are important for tumour regression in patients. Although various approaches to enhance the immunogenicity of tumour antigens have been described, we believe that an effective cancer vaccine must include tumour antigens that enable epitope spreading (7).

It should be noted that current bacterial expression methods are greatly limited in their ability to identify antigens that are posttranslationally modified. These modifications could play a key role in their immunogenicity. To circumvent this limitation, several eukaryotic expression systems such as baculovirus and retroviral surface display strategies have been established (8). Moreover, recent progress in proteomics has opened a new avenue for tumour antigen discovery. In this respect, Le Naour and colleagues have identified tumour-associated antigens through use of the proteomics-based approach followed by Western blotting with sera from cancer patients. This new method can probe the presence of autoantibodies against proteins carrying posttranslational modifications such as glycosylation and phosphorylation (10).

In order to develop a mucosal cancer vaccine, first we identified tumour antigens using a proteomics-based strategy. 
Second, tumour antigens were expressed on the surface of the $\mathrm{T} 7$ phage and their immunogenicity was investigated. Oral immunization of BALB/c mice with a recombinant $\mathrm{T} 7$ phage-displaying human Hsp27 inhibited tumour growth and metastasis of the 4T1 breast adenocarcinoma cell line.

\section{Materials and methods}

Cell culture and preparation of cell protein extracts. Mammary gland adenocarcinoma SK-BR-3 cells (ATCC HTB-30) were cultured in RPMI supplemented with $10 \%$ FCS and antibiotics. Normal human mammary epithelial cells (HMEC) (Clonetics/ BioWittaker) were cultured in complete MEGM medium (Clonetics/BioWittaker). Whole cell extracts were prepared from $5 \times 10^{7} \mathrm{SK}-\mathrm{BR}-3$ cells. The cells were washed twice with ice-cold PBS (phosphate-buffered saline), and lysed by adding $250 \mu 1$ 2D-lysis buffer (50 mM Tris- $\mathrm{HCl}$ pH 7.4, $0.3 \mathrm{M} \mathrm{NaCl}$, $3 \mathrm{mM}$ EDTA and $1 \%$ Triton $\mathrm{X}-100$ ) containing protease inhibitors. Subsequently, cell lysates were sonicated and then centrifuged at $15000 \mathrm{rpm} / \mathrm{min}$ for $5 \mathrm{~min}$ at $4^{\circ} \mathrm{C}$. The supernatants were used for immunoprecipitation (IP) with patients or healthy individual serum antibodies. Serum samples from patients with breast cancer or healthy individuals were obtained from Ullevål University Hospital (5). All sera were aliquoted and stored at $-70^{\circ} \mathrm{C}$ until use. With regard to animal studies, the murine breast carcinoma 4T1 cell line was purchased from ATCC and grown in RPMI medium supplemented with $10 \%$ FCS and antibiotics.

Immunoprecipitation and 2D gel electrophoresis. Protein-G Dynabeads (Dynal Biotech-Invitrogen) were washed 3 times with PBS and coated with either patient or healthy individual serum antibodies (100 $\mu 1$ serum, $50 \mu 1$ protein-G dynabeads). After incubation for $1 \mathrm{~h}$ at $4^{\circ} \mathrm{C}$, the beads were blocked for unspecific binding with BSA $(5 \mathrm{mg} / \mathrm{ml})$ for $1 \mathrm{~h}$ at $4^{\circ} \mathrm{C}$. Thereafter, the serum-coated beads were washed 3 times with 2D-lysis buffer and resuspended in $50 \mu 1$ 2D-lysis buffer. SK-BR3 whole cell extract ( $250 \mu 1)$ was added to the serumcoated beads and incubated overnight at $4{ }^{\circ} \mathrm{C}$ with agitation. The beads were washed three times with 2D-lysis buffer, once with 2D-lysis buffer containing $0.1 \%$ SDS (sodium dodecylsulphate), and then with 2D-lysis buffer to remove the SDS. The beads were then resuspended in $50 \mu 1$ of 2Dlysis buffer and incubated at $98^{\circ} \mathrm{C}$ for $10 \mathrm{~min}$. The beads were then captured on a magnet and the supernatant containing the immunoreactive proteins was collected. Protein samples (300 $\mu \mathrm{g} /$ per sample) were diluted in 2D-loading buffer $(6 \mathrm{M}$ Urea, 2 M Thiourea, 2\% Chaps, 0.3\% DTT, IPG-buffer pH 3$10,0.01 \%$ bromophenol blue) and then loaded onto linear IFG strips ( $\mathrm{pH} 3-10 ; 18 \mathrm{~cm}$, Amersham-Pharmacia). The proteins were separated according to their molecular weight on a vertical $8 \%$ SDS-PAGE using the PROTEAN II system (Bio-Rad). After electrophoresis, the protein spots were either visualized by silver-staining or transferred to nitrocellulose membrane using a Trans-blot semi-dry electrophoretic transfer cell system (Bio-Rad). Two gels were run simultaneously with equal amounts of the same proteins. One gel was silver-stained, whereas the other was used for Western blotting. The nitrocellulose membranes were incubated overnight at $4^{\circ} \mathrm{C}$ with patient or normal serum antibodies (1/500 dilution), washed and incubated with horseradish peroxidase (HRP)-conjugated anti-human IgG antibody (1/2000 dilution). Sera were diluted in PBS-Tween $20(1 \% \mathrm{v} / \mathrm{v})$ containing 1\% (v/v) non-fat dry milk. Immunodetection was performed with the ECL system. Differential protein spots were excised from the preparative gels, digested with trypsin and then identified by peptide mass fingerprinting using a MALDI-TOF mass spectrometer (ABI).

Expression of recombinant proteins in E. coli. The complete cDNA sequence of pyruvate dehydrogenase complex component X (PDHX) was predicted from the PDHX mRNA sequence (GenBank accession no. NM 003477.1). The full open reading frame was amplified by RT-PCR using firststrand cDNA prepared from SK-BR3 cells as a template. Primers to amplify the PDHX were forward 5'-ccagtgagaag gccgtca-3', and reverse 5'-gactaggcaagtcggatagga-3'. PCR products were directly ligated into pGEM-T eazy vector and positive clones were identified and confirmed by DNA sequencing. To express the protein, the open reading frame was PCR amplified using the following primers containing Bam $\mathrm{H} 1$ and Eco $\mathrm{R} 1$ restriction sites: 5'-aaaggatccaagatggcggc ctcc-3 and 5'-ttgaattcctaggcaagtcggat-3. The PCR product was digested with BamHI and EcoRI restriction enzymes and then ligated into Glutathione S-transferase (GST) fusion expression vector pGEX-6P-1 (Amersham). BL-21 E. coli cells were transformed with the PDHX-pGEX-6P-1 construct and positive colonies were verified by DNA sequencing. The fusion protein was purified according to the manufacturer's instructions and its purity was examined by SDS-PAGE. Protein samples were dialyzed in PBS prior to use.

Expression of tumour antigens in E. coli or the T7 phage system. In order to express tumour antigens, we PCR amplified several tumour antigens, including the Hsp27 detected in this study from cDNAs prepared from cancer cell lines. The forward and reverse primers used to RT-PCR amplify tumour antigens had the following sequences, respectively: Hsp27, 5'-aagaattctatgaccgagcgccgcgtc-3' and 5'-aagctttacttggcggcag tctcatcgga-3'; MelanA, 5'-cgggatccatgccaagagaagatgctcac-3' and 5'-cccaagcttttaaggtgaaataaggtggtggtgactgtt-3'; P53, 5'cgggatccatggaggagccgcagt- 3 ' and 5 '-cccaagcttcagtctgagtcagg ccctt-3'; MAGE1, 5'-cgggatccatcatgtctcttgagcaga-3' and 5'cccaagcttaactcatgctcagactccetctt-3'; RPL19, 5'-ccggatccatgag tatgctcaggct-3' and 5'-aatggctgatctatgtaagcttagaggccagtatgt-3'; SSX2, 5'-gccaaatacttctctaaggaagagtgg-3' and 5'- ttcactgttgtga acacttgcttcac-3'; and MAGEA9, 5'-agagtcatcatgcetcttgagc aga-3' and 5'- tgcaactcgtgctcagactcact-3'. The resulting PCR products were gel purified, digested with $B a m \mathrm{H} 1$ and HindIII restriction enzymes and then cloned in pQE-30 vector (Qiagen). The SSX2 and MAGE A9 were amplified with pfu DNA polymerase and then cloned into SmaI-cleaved pQE30 vector that was designed for cloning and expression of 6X His-tagged proteins that can be purified by immobilization on a metalchelating surface such as Ni-NTA HisSorb matrices. Based on the manufacturer's intructions, several tumour antigens were cloned, confirmed by DNA sequencing, expressed in E. coli M15 and then 6X His-tagged proteins were purified. To display the selected tumour antigens on the surface of the phage T7, the recombinant plasmids were digested with EcoRI and HindIII restriction enzymes and then DNA inserts were subcloned in 
T7Select EcoR1/HindIII arms as fusion proteins with the T7 10B capsid protein according to the manufacturer's instructions (Novagen). After in vitro packaging and plaque assays, the plaques were transferred to nitrocellulose and then immunoscreened with an anti-His mAb. Several positive clones were obtained and confirmed by automatic DNA sequencing (ABI prism 310 Genetic Analyzer).

Animal studies. Inbred female 7- to 9-week-old BALB/c mice were purchased from Taconic, Bomholt (Denmark) and maintained in a specific pathogen-free room at a constant temperature and humidity on a 12-h light and dark schedule with an air exchange rate of 20 changes/h. All animals were handled in accordance with the national legislation and institutional guidelines. BALB/c mice were immunized orally with either the wild-type phage or recombinant phage expressing Hsp27 (1011 TU/per immunization) once a week for 3 weeks. Immune responses were monitored by ELISA on day 26 following immunization. On day 30 after immunization, mice were subcutaneously injected with $4 \mathrm{~T} 1$ tumour cells $\left(5 \times 10^{6}\right.$ cells). The mice were kept under surveillance and when the tumour reached the maximum size in controls, mice were euthanized; on day 28 post cell inoculation. Tumour weights were determined for all groups. Also, the lungs were dissected and incubated in Bouins solution in order to visualize 4T1 metastases that were determined by vision under a light microscope as white spots, whereas the normal lung tissue appeared as yellow.

Enzyme-linked immunosorbent assay (ELISA). Patient serum antibodies reacting with recombinant tumour antigens were detected by ELISA. Briefly, 96-well microtiter plates were coated with recombinant proteins $(0.2 \mu \mathrm{g} /$ well $)$ in PBS buffer overnight at $4^{\circ} \mathrm{C}$. After blocking with PBS containing 5\% BSA for $1 \mathrm{~h}$ at room temperature (RT), the plates were washed and then incubated for $1 \mathrm{~h}$ at RT with serum samples $(1 / 500$ dilution in PBS/0.05\% Tween-20). Thereafter, the plates were washed 4 times with ELISA buffer and then incubated for $1 \mathrm{~h}$ with AP-conjugated anti-human IgG antibodies diluted 1/2500. The enzyme activity was detected with p-nitrophenyl phosphate disodium (Sigma) and absorbance was measured on an ELISA plate reader at $405 \mathrm{~nm}$. To characterize the immune responses induced by the phage-displayed antigens, ELISA plates were coated as above with either the recombinant phages or purified recombinant proteins and the detection was made with APconjugated anti-mouse $\operatorname{IgG} 1$ or $\operatorname{IgG} 2$ a diluted 1/2500.

\section{Results}

Identification of immunoreactive antigens. Despite extensive efforts that have been made in recent years to identify tumour antigens in human cancers (11), only a few have given some immune response when incorporated into cancer vaccines (3). Therefore, new tumour antigens and/or vaccine formulation are needed. To uncover new tumour antigens, we combined immunoprecipitation with a proteomics-based approach. In this protocol, serum antibodies from a breast cancer patient were immobilized on protein-G magnetic beads and used to immunoprecipitate potential tumour antigens from whole cell lysates prepared from the breast carcinoma cell line SKBR3.
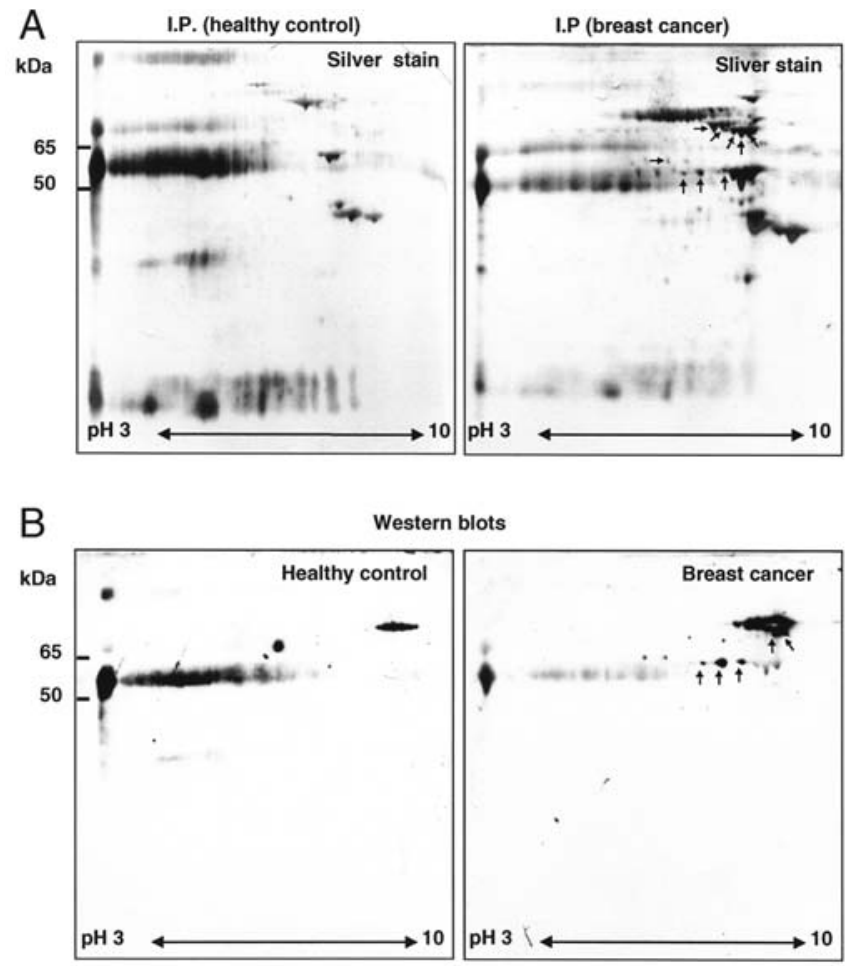

Figure 1. Representative 2D gel images of patient and normal serum immunoprecipitated SKBR3 proteins. (A) The immunoprecipitated proteins were separated on $\mathrm{pH}$ 3-10 nonlinear IPG strips in the first dimension, followed by $12 \%$ SDS-PAGE in the second dimension and then protein spots were visualized by silver-staining. (B) Representative results of Western blot analysis performed with serum from a patient with breast cancer or from a normal healthy female as indicated. Differentially immunoreactive spots (indicated by arrows) were identified by MALDI-TOF peptide mass fingerprinting (Table I).

Serum from a healthy female individual was used as a control to distinguish between the proteins that would represent false positive spots. After washing, the captured immunoreactive proteins were recovered from the beads and analysed by $2 \mathrm{D}$ gel electrophoresis followed by silver-staining (Fig. 1A, as a representative example). The samples were examined more than 3 times, and a significant number of patient serum-specific protein spots were detected consistently in each experiment. Subsequent to 2D gel electrophoresis, the proteins were transferred to nitrocellulose membranes and then incubated with serum antibodies from a breast cancer patient or healthy individual (Fig. 1B, as a representative example). Approximately 15 protein spots that showed specific reactivity with patient serum were excised, and mass spectrometry sequence was obtained from 9 spots (Table I). The expression of some of the identified proteins was reported to be increased and/or involved in breast cancer. For example, ADAM10, Hsp27 and aldolase A exhibited overexpression in different cancer types (12-14). While several mechanisms might account for the contribution of the identified proteins in cancer cell proliferation and survival, Hsps inhibited apoptosis by interacting with apoptotic molecules (13).

To confirm the immunoreactivity detected in 2D gel, PDHX protein was expressed in E. coli and Western blot analysis was performed with the purified GST-fusion protein (Fig. 2A). As shown in Fig. 2B, patient serum antibodies 
Table I. Identification of protein spots reacting with patient serum antibodies.

\begin{tabular}{ll}
\hline Protein & \multicolumn{1}{c}{ Name } \\
\hline 1 & Dihydrolipoamide S-acetyltransferase (DLAT) \\
2 & Pyruvate dehydrogenase protein X component (PDHX) \\
3 & B-ATPase F1 \\
4 & Hypothetical protein FLJ20361 \\
5 & Heat-shock protein-B 1 (Hsp27) \\
6 & ADAM10 \\
7 & Aldolase A \\
8 & Hydroxylmethylbilane synthase (HMBS) \\
9 & Vimentin \\
\hline
\end{tabular}
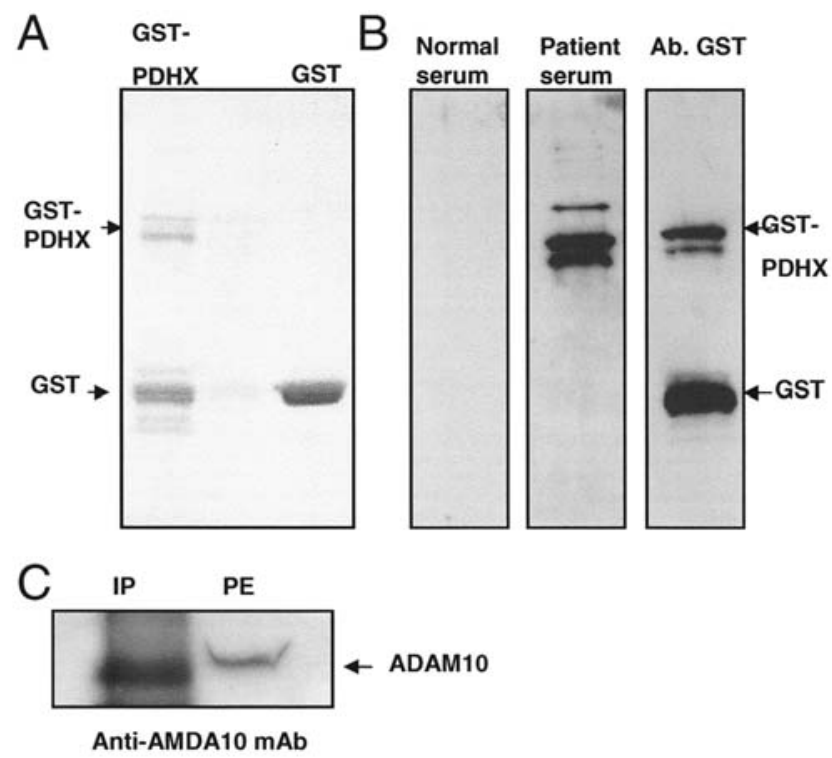

Figure 2. Expression and immunoreactivity of the recombinant PDHX expressed in E. coli. A) SDS-PAGE analysis of the GST-PDHX fusion protein. The gel was stained with Coomassie brillant blue. Lane 1, purified GST-PDHX fusion protein. Lane 2, purified GST protein. (B) Serum reactivity of the purified GST-PDHX fusion protein. The nitrocellulose membranes were incubated with serum antibodies from a patient with breast cancer or from a healthy female as indicated. As a control, the same preparation was incubated with an anti-GST monoclonal antibody. The GST-PDHX fusion protein was not stable, leading to the generation of GST protein as shown in panels A and B. (C) Protein lysates from SK-BR3 were immunoprecipitated (IP) using serum antibodies from a patient with breast cancer. Subsequently, immunoprecipitated proteins were separated on 10\% SDS-PAGE gel and then analysed by Western blotting using a commercially available antiADAM 10 monoclonal antibody. Protein extracts (PE) from SK-BR3 that express ADAM10 protein were used as positive control.

reacted with the fusion protein, whereas no reactivity was detected with normal serum antibodies. Moreover, an ELISA identified high-titer IgG antibodies in 4 out of 36 patients with breast cancer. Autoantibodies against PDHX were detected in patients with primary biliary cirrhosis (PBC), thus confirming the immunogenicity of the natural protein (15). Analysis of immunoprecipiated proteins with Western blotting using a monoclonal antibody against ADAM10 also confirmed its immunoreactivity with patient sera (Fig. 2C).

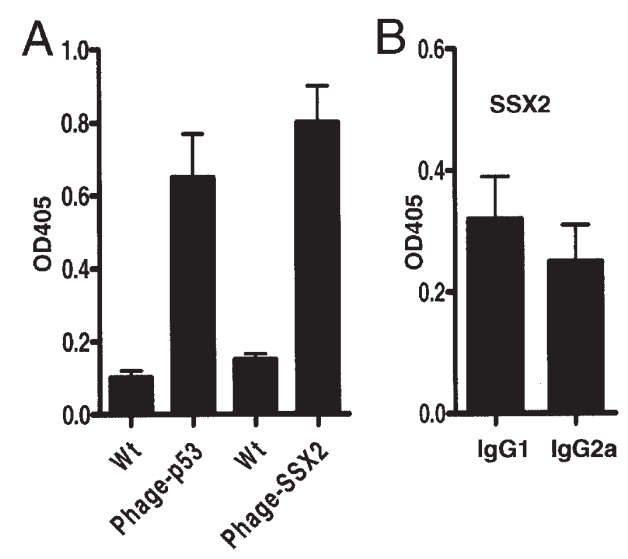

Figure 3. Immune response to phage-displayed tumour antigens. Groups of 3 mice were immunized orally with the T7 phage expressing p53 or SSX2 tumour antigen. As control, a group of 3 mice were immunized with only the wild-type phage. Serum samples were collected on day 28 post immunization and used in ELISA coated with purified p53 or SSX2 antigen (A). Sera were also analyzed for $\mathrm{IgG} 1$ and $\mathrm{IgG} 2 \mathrm{a}$ antibodies against the SSX2 antigen (B).

Tumour antigen expression on the surface of the phage. Previously, we have shown that recombinant phages can be used as a carrier for immunization, and therefore as a tool for vaccine development (16). Indeed, we have shown that the B cell epitopes displayed on the surface of the phage can induce a strong immune response against the displayed peptides in $\mathrm{BALB} / \mathrm{c}$ mice (16). Also, we have shown that phage-expressing $\mathrm{B}$ cell epitopes can be used in high-throughput format to probe humoral immune responses in patients with breast cancer (17). Therefore, in this study we hypothesized that oral immunization with phage-displayed tumour antigens could lead to the induction of effective immune responses against the expressed antigens. To achieve this goal, several tumour antigens, including SSX2, MAGE-1 and p53 (3), were expressed on the surface of the T7 phage as described in the Materials and methods. Mice orally immunized with the T7-recombinant phages developed $\mathrm{IgG}$ responses against the displayed tumour antigens as illustrated in Fig. 3A. Both Th1 and Th2 responses were induced against the recombinant proteins (Fig. 3B).

To test the therapeutic potential of the developed T7 recombinant phages, we used Hsp27 as an antigen model. Hsp27 is a homologue of Hsp25 that was found to be expressed on the surface of 4T1 breast carcinoma cells (18). The recombinant protein was expressed as fusion with the 10B capsid protein. Subsequent to cloning and plaque array immunoscreening, several positive clones were obtained and verified by DNA sequencing. The expression of Hsp27 on the surface of the phage was established by using commercially available polyclonal antibodies against Hsp27 (Fig. 4A). In contrast to the wild-type phage, the recombinant phage showed a strong immunoreactivity.

Oral immunization with the recombinant phage. In contrast to several routes of immunization, oral administration of antigens is an attractive option for mucosal immunisation. BALB/c mice were orally immunized with either the recombinant Hsp27 phage or wild-type phage $\left(10^{11} \mathrm{TU} / 500 \mathrm{ml}\right)$ on days $0,8,16$ and 24. Immune responses against the phages were determined on day 28 . The animals responded to the recombinant phages 

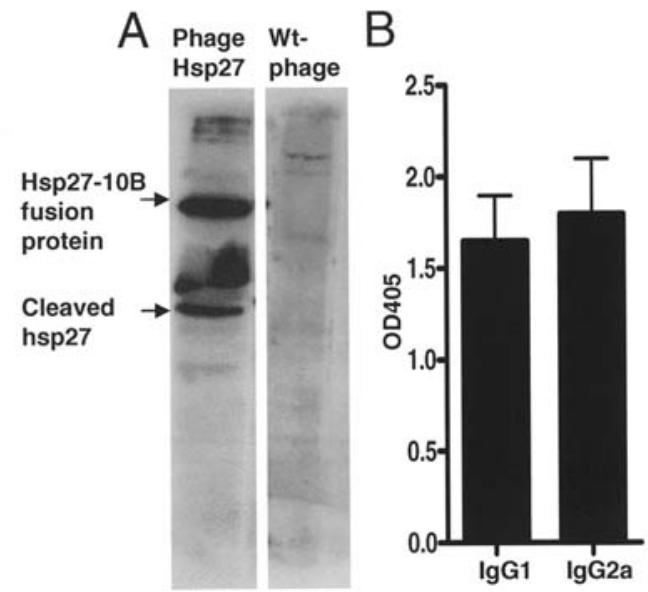

Figure 4. Expression of Hsp27 on T7 phage. The human Hsp27 was cloned in-frame with the $\mathrm{T} 7 \mathrm{10B}$ protein such that the recombinant fusion protein is displayed on the surface of the phage. (A) Western blot analysis of the wild-type (Wt) phage and a T7 phage displaying Hsp27. Membranes were incubated with an anti-Hsp27 polyclonal antibody and the detection was made with HRP-conjugated anti-rabbit IgG and the ECL reagent. (B) A group of 7 mice were immunized with recombinant T7 phage particles. Serum samples were collected on day 28 post immunization and then the levels of phage-specific IgG1 and IgG2a were determined by ELISA.

as well as to the Hsp27 proteins expressed by 4T1 tumour cells. To characterise antibody responses against the recombinant phages, the levels of antigen-specific IgG subclasses IgG1 and IgG2a were assessed by ELISA (Fig. 4B). Orally immunized mice developed balanced Th1 and Th2 type responses. Notably, Th1 cells stimulate $\operatorname{IgG} 2 \mathrm{a}$ antibody production regulated by IFN- $\alpha$, whereas IgG1 antibodies are induced under control of the Th2 cytokine IL4.

Oral immunization blocked tumour growth and metastasis. Hsps are evolutionarily highly conserved proteins and are abundantly expressed in both prokaryotic and eukaryotic organisms (19). Previous studies have shown that certain cancer cells, including the murine 4T1 breast carcinoma cell line, can express high levels of Hsps on their surface (18). The 25-kDa Hsp (Hsp 25) belongs to the family of small Hsps and is the murine homologue of human Hsp27. To assess the effects of mucosal immune responses on tumour growth, we have used the 4T1 mouse tumour model. When inoculated into BALB/c mice, 4T1 spontaneously produces local tumours that can metastasize to various tissues, including the lung, liver and lymph nodes. Mice were subcutaneously injected with 4T1 tumour cells $\left(5 \times 10^{6}\right.$ cells) on day 30 after immunization following oral immunisation with either the $\mathrm{T} 7$ phage expressing the Hsp27 or wild-type phage. All mice that orally received the wild-type phage developed tumours within 2 weeks, with tumours increasing and reaching $2.5 \pm 0.5 \mathrm{~g}$ within 4 weeks after cell inoculation (Fig. 5A). In contrast, mice immunized with $\mathrm{T} 7$ phage expressing the Hsp27 developed tumours that had a significantly smaller average weight versus the wild-type phage $(1.5 \pm 0.2 \mathrm{~g}, \mathrm{p}<0.05)$. Also, the number of lung metastases in mice immunized with the wild-type phage was significantly higher than that seen in mice immunized with recombinant phage $(80 \pm 15$ vs $40 \pm 10, \mathrm{p}<0.05)$ (Fig. 5B). Collectively, these data indicate for the first time that a recombinant $\mathrm{T} 7$
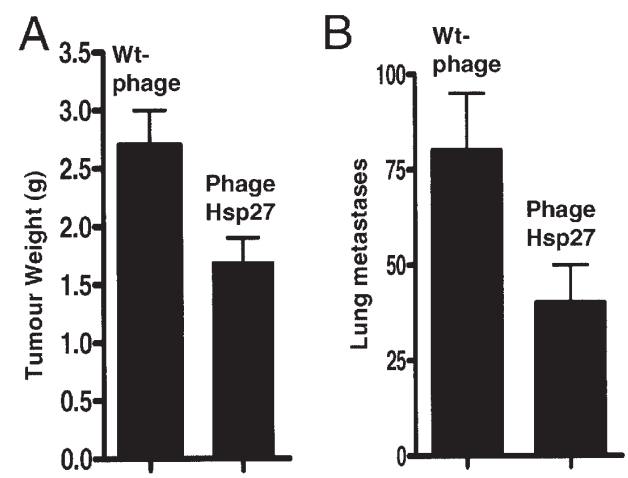

Figure 5. Oral administration of recombinant T7 phage inhibited tumour growth. Groups of 7 BALB/c mice were orally immunized with Hsp27 recombinant phage or wild-type $(\mathrm{Wt})$ phage. At approximately four weeks post immunization, mice were subcutaneously inoculated with 4T1 cancer cells. On day 28 post cell inoculation, all animals were euthanized and endpoint tumour weights (A) and lung cancer metastases were determined (B). Lung metastases were analysed in 3 mice/group.

phage stably expressing Hsp27, a homologue of murine Hsp25, is able to inhibit outgrowth of 4T1 cells expressing Hsp25 in a therapeutic setting. We also found that immunization with lactic acid bacteria expressing tumour antigens inhibited tumour growth (unpublished data).

\section{Discussion}

In the present study, we have implemented a proteomics-based approach to identify tumour antigens and demonstrated that non-pathogenic phages can function as a carrier for mucosal vaccination against tumours, thus highlighting their clinical potential in future cancer vaccines. Moreover, the adjuvant property of the phages seems to induce a balance between Th1 and Th2 type responses that might be required for tumour eradication.

New biomarkers identified by serological screening, once validated, should have utility in cancer screening, diagnosis or prognosis. Some of the identified tumour antigens have been involved in cancers. The ADAM proteins are multifunctional, membrane-bound cell surface glycoproteins, and more than 30 different members have been characterized to date (12). In addition, they are differentially expressed in malignant tumours and may therefore participate in the pathology of carcinomas. Increased expression of ADAM10 was detected in gastric cancer and human breast cancer (12). The heat shock proteins belong to the molecular chaperone family which includes a diverse group of evolutionarily conserved proteins with a wide range of cellular activities, including the regulation of intracellular homeostasis, protein stabilization, and enhancement of cell survival after exposure to environmental stressors (19). Enhanced Hsp27 expression has been found in several human tumours including those of the stomach, breast, and prostate (13). Also, autoantibodies to Hsp90 were found in several cancer types, including breast and ovarian carcinoma (20).

Because of the weak clinical effectiveness of current cancer vaccines, new vaccine formulations and/or vaccination methods are urgently needed. Previously, we have shown that xenogeneic immunization with glioma membrane proteins can block glioma tumour growth in syngeneic rats (21). Due to 
their high sequence homology, Hsps might be a potential candidate for molecular mimicry and therefore could act as potential autoantigens. Previous studies have demonstrated the presence of anti-Hsp antibodies in autoimmune inflammatory responses, such as SLE and rheumatoid arthritis, in which the initiating antigenic stimulus is more likely a pathogen (22). As illustrated in this study, we have shown that mucosal immunization with a recombinant phage displaying on its surface human Hsp27 can induce an immune response capable of inhibiting tumour growth of mouse 4T1 breast carcinoma. It should be noted that no adverse effects were detected in human volunteers that received phage T4 orally (23). Therefore, the use of phages as carriers for tumour antigens should be feasible in patients with cancer. If necessary, phages can be irradiated to inactivate their genetic materials.

Using basic molecular biology techniques, phages and bacteria can be engineered to display specific $\mathrm{T}$ cell epitopes on their surface along with effector cytokines to direct either Th1 or Th2 type responses. Previous studies have shown that attenuated and commensal microorganisms can be used as carriers for vaccination (24). In this regard, Poo and colleagues demonstrated that the expression of E7 protein of human papillomavirus type 16 on the surface of Lactobacillus case $i$ can induce E7-specific antitumour effects in C47/BL6 mice (25). Oral immunization with Salmonella typhimurium expressing the NY-ESO-1 antigen also inhibited tumour growth in mice (26). In collaboration with Professor Eijsink's group (Norwegian University of Life Science, Ås), we have chosen Lactobacillus plantarum to express tumour antigens. This probiotic bacterium is present in human gastrointestinal tract and used in several food products (27). Our data show that Lactobacillus plantarum expressing a variety of tumour antigens on its surface can induce a mucosal Th- 1 immune response capable of inhibiting tumour growth in mice (unpublished data).

To effectively activate immune responses against tumour cells, at least three separate types of signals are required. The first signal must be directed at the innate immune system, whereas the other two are directed at the adaptive immune system. The activation of dendritic cells represents the first major signal that can be induced by conserved pathogen associated molecular patterns (PAMPs) on microbes via Tolllike receptors (TLRs) expressed by immune cells, in particular dendritic cells (DCs) (28). Through the recognition of PAMPs the innate immune system is capable of discriminating between self and non-self. Phages and bacteria can directly activate DCs via surface TLRs or other cytoplasmic detection receptors (e.g. NOD proteins), and therefore initiate specific signalling pathways that lead to distinct immune responses. Signalling via TLRs induces the production of proinflammatory cytokines and chemokines as well as the overexpression of costimulatory and MHC molecules on DCs. Activated DC cells would then acquire tumour antigens expressed by the phages or bacteria that they would then process and present on their surface. Once activated, DCs migrate to the nearest draining lymph nodes where they can present antigens derived from pathogens to naïve $T$ cells and induce differentiation of these cells by secreting appropriate cytokines. In this process, DC and T cells establish main contacts via TcR/MHC and CD28/CD80/86 interacting molecules (signal 2 and 3 , respectively). Activated tumour-specific T cells will eventually migrate back to tumours to provide help to other immune cells and/or kill malignant cells. In the case of mucosal vaccination, intestinal mucosal DCs take-up phages or bacteria in either the lamina propria or the Peyer's patches. Then activated dendritic cells migrate into the mesenteric lymph node where they activate $\mathrm{T}$ cells and induce plasma cells that produce $\operatorname{IgG}$ or $\operatorname{IgA}$, which home to the mucosa or other locations (29).

Most mucosal surfaces are populated by commensal microorganisms that provide many benefits to the mammalian host (30). How then do mucosal DCs distinguish commensal bacteria from those expressing tumour antigens or from pathogenic bacteria? In other words how are the commensal bacteria ignored by the immune system? Do the virulence factors expressed by pathogenic bacteria contribute to the activation of mucosal DCs? Whatever the mechanisms of mucosal DC activation by bacteria or phage expressing antigens, it is important to note that $\mathrm{M}$ cells are specialized epithelial cells in gut-associated lymphoid tissues (GALT) that transport bacteria and antigens to the underlying immune cells, thereby facilitating immune responses (31). Therefore, immune response to phage and bacteria might dependent on the ability of these microorganisms to be transported across the lumen. The more we learn about how mucosal vaccines inhibit tumour growth, the strategies employed to design successful mucosal cancer vaccines are likely to become even more sophisticated. For example, strain selection and antigens that can cross the lumen without being degraded might improve the vaccine. Also, the vaccination routes should take into account cancer types. For example, in the case of lung and vaginal tumours, the vaccine could be delivered via nasal or vaginal route, respectively. In addition to being a carrier for mucosal vaccination, phage-displayed tumour antigens can be useful in profiling immune response in patients. In this respect, we have shown that recombinant phages can be used in high-throughput format to probe humoral immune response in patients with breast cancer (17). This simple serological test could provide useful diagnostic markers for the detection of tumour antigens in cancer patients. Using sera from patients with breast cancer, we were able to show specific reactivity to tumour antigens expressed on the surface of the T7 phage. Collectively, the present study highlights the diverse applications of phage and bacterial display strategies with regard to serological profiling and vaccination.

\section{Acknowledgements}

This study was supported in part by the gene therapy program at the Norwegian Radium Hospital to M. Sioud. We thank Anders $\emptyset$ verbye for his help with MALDI-TOF analysis.

\section{References}

1. van der Bruggen $\mathrm{P}$, Traversari C, Chomez $\mathrm{P}$, Lurquin $\mathrm{C}$, De Plaen E, Van den Eynde B, Knuth A and Boon T: A gene encoding an antigen recognized by cytolytic $\mathrm{T}$ lymphocytes on a human melanoma. Science 254: 1643-1647, 1991.

2. Sahin U, Türeci Ö, Schmitt H, Cochlovius B, Johannes T, Schmits R, Stenner F, Luo G, Schobert I and Pfreundschuh M: Human neoplasms elicit multiple specific responses in the autologous host. Proc Natl Acad Sci USA 92: 11810-11813, 1995. 
3. Jäger D: Potential target antigens for immunotherapy identified by serological expression cloning (SEREX). Methods Mol Biol 360: 319-326, 2007.

4. Dybwad A, Førre $\varnothing$, Kjeldsen-Kragh J, Natvig JB and Sioud M: Identification of new cell epitopes in the sera of rheumatoid arthritis patients using a random nanopeptide phage library. Eur J Immunol 23: 3189-3193, 1993.

5. Hansen MH, Østenstad B and Sioud M: Antigen-specific IgG antibodies in stage IV long-time survival breast cancer patients: Mol Med 7: 230-239, 2001.

6. Sioud M: How does autoimmunity cause tumor regression? A potential mechanism involving cross-reaction through epitope mimicry. Mol Med 8: 115-119, 2002.

7. Hansen MH, Østenstad B and Sioud M: Identification of immunogenic antigens using a phage-displayed cDNA library from an invasive ductal breast carcinoma tumour. Int J Oncol 19: 1303-1309, 2001.

8. Boder ET and Wittrup KD: Yeast surface display for screening combinatorial polypeptide libraries. Nat Biotechnol 15: 553-557, 1997.

9. Le Naour F, Misek DE, Krause MC, et al: Proteomic-based identification of RS/DJ-1 as a novel circulating tumour antigen in breast cancer. Clin Cancer Res 11: 3328-3335, 2001.

10. Le Naour F: Identification of tumor antigens by using proteomics. Methods Mol Biol 360: 327-334, 2007.

11. Sioud $M$ and Hansen MH: Profiling the immune response in patients with breast cancer by phage-displayed cDNA libraries. Eur J Immunol 31: 716-725, 2001.

12. Lendeckel U, Kohl J, Arndt M, Car-McGrath S, Donat H and Röcken C: Increased expression of ADAM family members in human breast cancer and breast cancer cell lines. J Cancer Res Clin Oncol 131: 41-48, 2005.

13. Jolly $\mathrm{C}$ and Morimoto RI: Role of the heat shock response and molecular chaperones in oncogenesis and cell death. J Natl Cancer Res Inst 92: 1564-1572, 2000.

14. Asaka M, Kimura T, Meguro T, Kato M, Kudo M, Miyazaki T and Alpert E: Alteration of aldolase isozyme in serum and tissues of patients with cancer and other diseases. J Clin Lab Anal 8: 144-148, 1994

15. Melegh B, Skuta G, Pajor L, Hegedus G and Sumegi B: Autoantibodies against subunits of pyruvate dehydrogenase and citrate synthase in a case of paediatric biliary cirrhosis. Gut 42 : 753-756, 1998.

16. Sioud M, Dybwad A, Jespersen L, Suleyman S, Natvig JB and Førre $\varnothing$ : Characterization of naturally occurring autoantibodies against tumour necrosis factor-alpha (TNF- $\alpha$ ): in vitro function and precise epitope mapping by phage epitope library. Clin Exp Immunol 98: 520-525, 1994.
17. Cekaite L, Haug O, Myklebost O, Aldrin M, Østenstad B, Holden M, Frigessi A, Hovig E and Sioud M: Analysis of the humoral immune response to immunoselected phage-displayed peptides by a microarray-based method. Proteomics 4: 2572-2582, 2004.

18. Bausero MA, Page DT, Osinaga E and Asea A: Surface expression of Hsp25 and Hsp72 differentially regulates tumour growth and metastasis. Tumor Biol 25: 243-251, 2004.

19. Soti CS and Csermely P: Molecular chaperones in the etiology and therapy of cancer. Pathol Oncol Res 4: 316-321, 1998.

20. Luo LY, Herrera I, Soosaipillai A and Diamandis EP: Identification of heat shock protein 90 and other proteins as tumour antigens by serological screening of an ovarian carcinoma expression library. Br J Cancer 87: 339-343, 2002.

21. Sioud M and Sørensen DR: Generation of an effective anti-tumor immunity after immunization with xenogeneic antigens. Eur J Immunol 33: 38-45, 2003.

22. Corrigall VM and Panayi GS: Autoantigens and immune pathways in rheumatoid arthritis. Crit Rev Immunol 22: 281-293, 2002.

23. Bruttin A and Brüssow H: Human volunteers receiving Escherichia coli phage T4 orally: a safety test of phage therapy. Antimicrob Agents Chemother 49: 2874-2878, 2005.

24. Medina E and Guzmán CA: Use of live bacterial vaccine vectors for antigen delivery: potential and limitation. Vaccine 19: 1573-1580, 2001

25. Poo H, Pyo H-M, Lee T-Y, Yoon S-W, Lee J-S and Kim C-J: Oral administration of human papillomavirus type 16 E7 displayed on Lactobacillus casei induces E7-specific antitumour effects in C57/BL6 mice. Int J Cancer 119: 1702-1709, 2006.

26. Nishikawa H, Sato E, Briones G, et al: In vivo antigen delivery by a Samonella typhimurium type III secretion system for therapeutic cancer vaccines. J Clin Invest 116: 1946-1954, 2006.

27. Seegers JF: Lactobacilli as live vaccine delivery vectors: progress and prospects: Trends Biotechnol 20: 508-515, 2002.

28. Akira S, Takeda $\mathrm{K}$ and Kaisho T: Toll-like receptors: critical proteins linking innate and acquired immunity. Nat Immunol 2: 675-680, 2001.

29. Kelsall BL and Leon F: Involvement of intestinal dendritic cells in oral tolerance, immunity to pathogens, and inflammatory bowel disease. Immunol Rev 206: 132-148, 2005.

30. Hooper LV, Midtvedt T and Gordon JI: How host-microbial interactions shape the nutrient environment of the mammalian intestine. Ann Rev Nutr 22: 283-307, 2002.

31. Neutra MR, Pringault RE and Kraehenbuhl JP: Antigen sampling across epithelial barriers and induction of mucosal immune responses. Annu Rev Immunol 14: 275-300, 1996 